\title{
Improved Quantum Particle Swarm Optimization for Mangroves Classification
}

\author{
Zhehuang Huang ${ }^{1,2}$ \\ ${ }^{1}$ Cognitive Science Department, Xiamen University, Xiamen 361005, China \\ ${ }^{2}$ School of Mathematics Sciences, Huaqiao University, Quanzhou 362021, China \\ Correspondence should be addressed to Zhehuang Huang; tingqian3@163.com
}

Received 20 October 2014; Revised 12 February 2015; Accepted 12 February 2015

Academic Editor: Xue Cheng Tai

Copyright (c) 2016 Zhehuang Huang. This is an open access article distributed under the Creative Commons Attribution License, which permits unrestricted use, distribution, and reproduction in any medium, provided the original work is properly cited.

\begin{abstract}
Quantum particle swarm optimization (QPSO) is a population based optimization algorithm inspired by social behavior of bird flocking which combines the ideas of quantum computing. For many optimization problems, traditional QPSO algorithm can produce high-quality solution within a reasonable computation time and relatively stable convergence characteristics. But QPSO algorithm also showed some unsatisfactory issues in practical applications, such as premature convergence and poor ability in global optimization. To solve these problems, an improved quantum particle swarm optimization algorithm is proposed and implemented in this paper. There are three main works in this paper. Firstly, an improved QPSO algorithm is introduced which can enhance decision making ability of the model. Secondly, we introduce synergetic neural network model to mangroves classification for the first time which can better handle fuzzy matching of remote sensing image. Finally, the improved QPSO algorithm is used to realize the optimization of network parameter. The experiments on mangroves classification showed that the improved algorithm has more powerful global exploration ability and faster convergence speed.
\end{abstract}

\section{Introduction}

Quantum particle swarm optimization algorithm (QPSO) is a new evolutionary algorithm proposed in $2005[1,2]$. Researchers found that the human learning process has great uncertainty which is very similar to the quantum behaviour of particle, so each individual can be described as a particle in quantum space. In recent years, a series of papers have focused on the application of QPSO, such as financial forecasting [3], sensor array [4], clinical disease diagnoses [5], classification and clustering [6], fuel management optimization [7], feature selection [8], and other areas $[9,10]$. But traditional QPSO is easy to fall into local optimum value and the convergence rate is slow. How to avoid prematurity and maintain fast convergence rate at the same time is a major problem. In order to improve the performance of QPSO, researchers have made some attempts. Sun et al. [11] reported a global search strategy of quantum behaved particle swarm optimization. In [12], chaotic mutation operator is introduced to quantum particle swarm optimization, instead of random sequences in QPSO; chaotic mutation operator is a powerful strategy to diversify the QPSO population and can improve the performance in preventing premature convergence to local minima. In [13], an improved quantum particle swarm optimization algorithm based on real coding method is presented which can improve the performance of QPSO.

Mangrove ecosystems play irreplaceable and important roles for the stabilization and equilibrium of coastal estuary as a unique ecosystem of land and sea [14-17]. Grasping the status of mangrove communities timely and accurately can provide important information for the protection and restoration of mangrove ecosystems. But the spatial resolution of TM images is not high and the spectral similarity of mangrove communities is strong [18-20]. Due to these problems, it is necessary to adopt a more intelligent approach to improve the accuracy of mangrove classification.

We can use synergetic neural network (SNN) proposed by Haken [21] to realize intelligent mangroves classification. The basic principle of synergetic neural network is that the pattern recognition procedure can be viewed as the competition progress of many order parameters. One advantage 
of synergetic neural network method is robustness against noise and occlusion; using this method we will be able to better handle fuzzy matching of mangroves classification whose contextual information is not complete. Synergetic pattern recognition method has been successfully used in face recognition [22], automatic control field [23, 24], and exon recognition [25]. Mangroves classification can also be considered as a problem of pattern recognition, and it is also entirely possible to use this method to solve mangroves classification. At present, the mainstream studies of SNN focus on the selection of prototype pattern vector [26], setting of attention parameter [27-29], reconstruction algorithm of order parameters [30], and so on. The network parameters directly influence the synergetic recognition performance. The adjustment of network parameters is a global behavior and has no general research theory to control the parameters in the recognition process at present. In this paper, we proposed an improved algorithm which can effectively choose network parameters of SNN.

This paper is organized as follows. An improved QPSO algorithm is presented in Section 2. In Section 3, mangroves classification model based on synergetic neural network is introduced. An improved SNN model based on improved QPSO is presented in Section 4. In Section 5, some experimental results and conclusions are given.

\section{Improved QPSO Based on Adaptive Behavior Selection (AQPSO)}

In this section, we present an improved QPSO. First, diversity function is employed as feature functions. Second, adaptive movement behavior is introduced into quantum particle swarm optimization for the first time.

2.1. Diversity Function. Diversity function is used to describe the dispersion degree of particles. Diversity function $g_{1}(t)$ describes adaptive diversity, where $t$ refers to the current iteration number. Consider

$$
D(t)=\frac{\min \left(f_{\mathrm{gb}}, f_{\mathrm{avg}}^{t}\right)}{\max \left(f_{\mathrm{gb}}, f_{\mathrm{avg}}^{t}\right)}, \quad g_{1}(t) \in(0,1], i=1,2, \ldots, n,
$$

where $f_{\text {avg }}^{t}$ means the average fitness value of the $i$ th iterative and $f_{\mathrm{gb}}$ is current global optimal value. $g_{1}(t)=1$ means the diversity is poor. $g_{1}(t) \ll 1$ means the diversity is good.

2.2. Adaptive Movement Behavior. From the QPSO algorithm, we can know local attractor $q$ is determined by local optimum pbest and global optimum gbest:

$$
q=\varphi \cdot \text { pbest }+(1-\varphi) \cdot g \text { best }
$$

where $\varphi=c_{1} r_{1} /\left(c_{1} r_{1}+c_{2} r_{2}\right), r_{1} \sim U(0,1), r_{2} \sim U(0,1)$.

In standard QPSO algorithm, $c_{1}$ and $c_{2}$ are employed as two accelerating factors which not only will affect convergence speed, but also may lead to the occurrence of premature phenomenon. In the early iterations, the current position and the optimal location of particles are far away. At later stage, the current position and the optimal location are closer. To effectively avoid the occurrence of premature phenomenon, $D(t)$ can be used to describe the closer degree between particles and the global optimal position, so as to select the corresponding acceleration factor:

$$
\begin{aligned}
& c_{1}= \begin{cases}(M c-m c) \times \tan \left(\frac{\mathrm{MAXITER}-t}{\mathrm{MAXITER}}\right)+m c, & D(t)>R_{1}, \\
(M c-m c) \times \frac{\text { MAXITER }-t}{\mathrm{MAXITER}}+m c, & D(t) \leq R_{1},\end{cases} \\
& c_{2}= \begin{cases}(M c-m c) \times \frac{\text { MAXITER }-t}{\mathrm{MAXITER}}+m c, & D(t)>R_{1}, \\
(M c-m c) \times \tan \left(\frac{\text { MAXITER }-t}{\text { MAXITER }}\right)+m c, & D(t) \leq R_{1} .\end{cases}
\end{aligned}
$$

2.3. The Improved Algorithm. The improved QPSO algorithm is shown as follows.

(1) Initialize variables.

(2) Generate initial artificial particle swarm.

(3) Update the local optimum position $P_{i j}$ and the global best position $P_{g i}$.

(4) Each particle updates its location through different behavior.

(5) Perform adaptive movement behavior by (3).

(6) The threshold gradually reduced which can lead to the dispersion decreasing of particles.

(7) Record the optimal value.

(8) If the iteration is terminated, output the optimal value; otherwise return to Step (3).

\section{An Improved SNN Model Based on AQPSO}

3.1. Mangroves Classification Based on SNN Model. SNN model is a top-down network constructed by synergetics different from traditional neural network, and it does not produce the pseudostate. An unrecognized pattern, $q$, is constructed by a dynamic process which translates $q$ into one of the prototype pattern vectors $v_{k}$ through status $q(t)$; namely, this prototype pattern is closest to $q(0)$. The process is described as in the following equation: $q \rightarrow q(t) \rightarrow v_{k}$.

A dynamic equation can be given for unrecognized pattern:

$$
\dot{q}=\sum_{k=1}^{M} \lambda_{k} v_{k}\left(v_{k}^{+} q\right)-B \sum_{k^{\prime} \neq k}\left(v_{k^{\prime}}^{+} q\right)^{2}\left(v_{k}^{+} q\right) v_{k}-C\left(q^{+} q\right) q+F(t)
$$

where $q$ is the status vector of input pattern with initial value $q_{0}, \lambda_{k}$ is attention parameter, $v_{k}$ is prototype pattern vector, and $v_{k}^{+}$is the adjoint vector of $v_{k}$. 


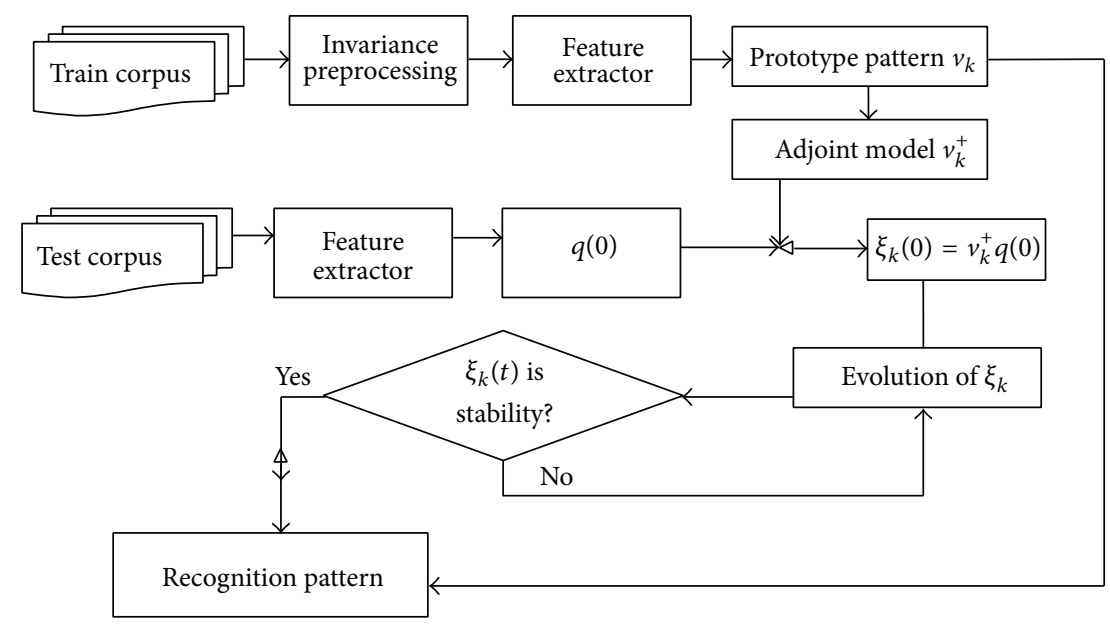

Figure 1: The flow chart of recognition process.

Corresponding dynamic equation of order parameters is

$$
\dot{\xi}_{k}=\lambda_{k} \xi_{k}-B \sum_{k^{\prime} \neq k} \xi_{k^{\prime}}^{2} \xi_{k}-C\left|\sum_{k^{\prime}=1}^{M} \xi_{k^{\prime}}^{2}\right| \xi_{k}
$$

where $\xi_{k}$ satisfies initial condition

$$
\xi_{k}=v_{k}^{+} q(0)
$$

A method for mangroves classification using synergetic neural network technique is presented, as shown in Figure 1.

The mangrove classification model based on SNN mainly includes the following steps.

Step 1. Extract feature from training corpus and calculate prototype pattern vectors which satisfied the condition of normalization and zero-mean.

Step 2. Obtain the adjoint pattern of the prototype patterns.

Step 3. Extract feature from test corpus and calculate test pattern vectors $q_{k}(0)(k=1,2, \ldots, N)$ which satisfied the condition of normalization and zero-mean.

Step 4. Calculate the initial values of order parameters according to (6).

Step 5. Evolution equation is performed for the competition among order parameters according to (5).

Step 6. If the values of $\xi_{k}$ in the evolution can reach stable condition, output the final results; otherwise continue performing evolution equation in Step 5.

3.2. Parameters Optimization of SNN Based on AQPSO. The network parameters of synergetic neural network directly influence the synergetic recognition performance. There is no general research theory to control the parameters in the recognition process at present. How to construct network parameter and choose a more efficient optimization method is an important task. To solve these problems, AQPSO is used in this section to effectively select network parameters.

A parameters optimization of synergetic neural network based on AQPSO is shown as Figure 2. Firstly, we can reconstruct the attention parameter $\lambda_{k}$ based on the measuring similarity between the prototype pattern $v_{k}$ and the test pattern $q_{l}$ :

$$
\begin{gathered}
\lambda_{k}=\alpha \cos \left(v_{k}, q_{l}\right), \\
\cos \left(v_{k}, q_{l}\right)=\frac{v_{k} \cdot q_{l}}{\left|v_{k}\right|\left|q_{l}\right|} .
\end{gathered}
$$

Secondly, AQPSO algorithm is used to search the global optimum parameters of network $(\alpha, B, C)$ in the corresponding parameter space.

The reconstruction and optimization of network parameters based on AQPSO can be described as follows.

(1) Obtain feature vectors from train corpus and test corpus and construct prototype pattern $v_{k}(k=$ $1,2, \ldots)$ and test pattern $q_{l}(l=1,2, \ldots)$.

(2) Calculate initial order parameter $\xi_{l k}$ according to (6).

(3) Set $\lambda_{k},(k=1,2, \ldots, m)$ according to (7).

(4) Optimization algorithms are used to search the global optimum parameters $(\alpha, B, C)$ of SNN in the corresponding parameter space.

(5) Get best mangrove categories through the evaluation of order parameter equation (5).

\section{Experiment}

In the experiment, we take 10 mangrove images as training samples. The size of images is $320 \times 320$. The categories of the mangrove images are shown as Table 1.

The prototype pattern of training samples is shown in Figure 3.

The corresponding adjoint mode $v_{k}^{+}$is shown in Figure 4.

In the next section, we use different mangrove images to test the performance of our proposed model. 


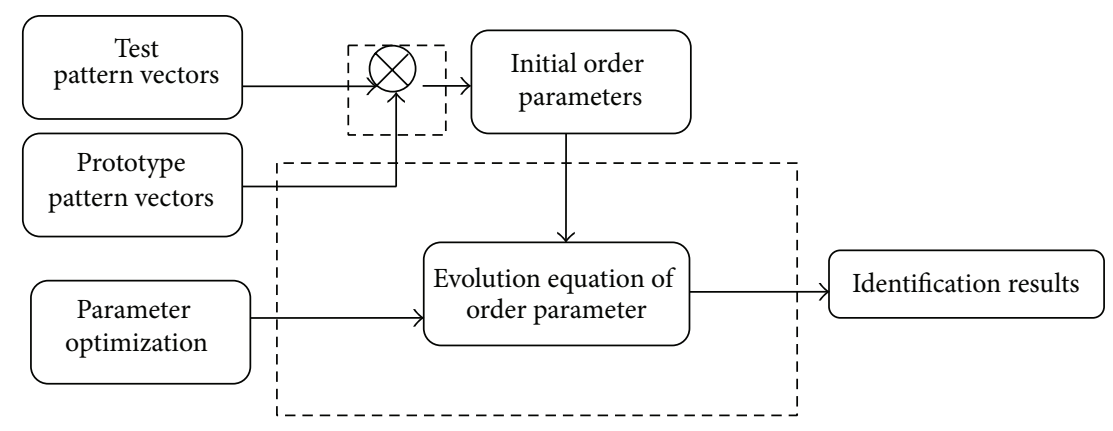

FIGURE 2: Parameters optimization of SNN based on AQPSO algorithm.
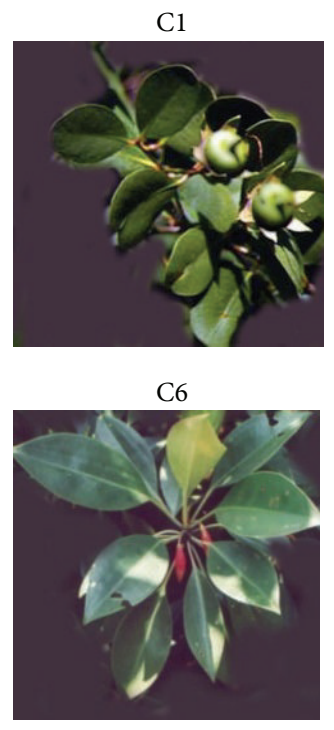

C2

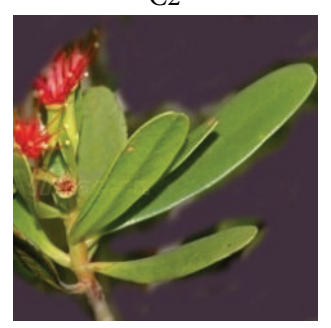

C7

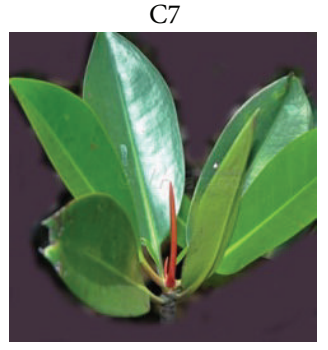

C3

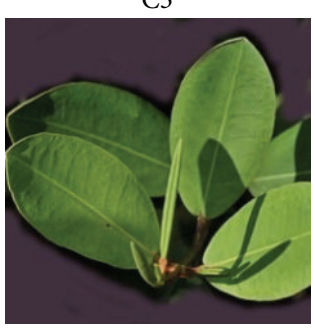

C8

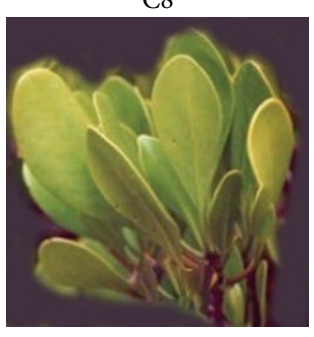

C4

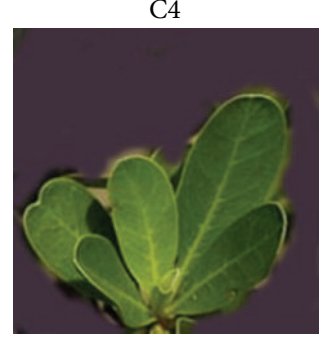

C9

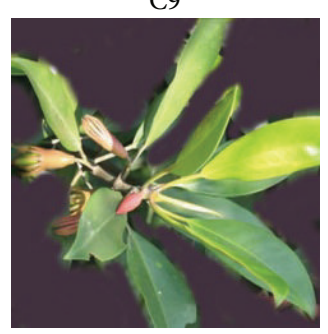

C5

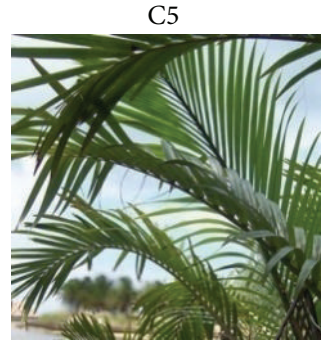

C10

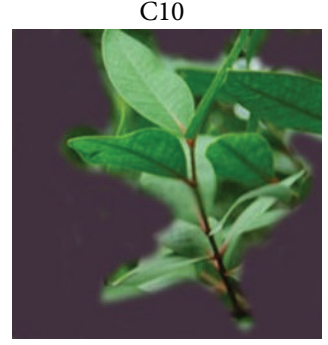

FIGURE 3: The corresponding prototype pattern of training samples.

TABle 1: Mangrove categories.

\begin{tabular}{lc}
\hline Class name & Mangrove \\
\hline C1 & Avicennia \\
C2 & Lumnitzera \\
C3 & Pelliciera \\
C4 & Conocarpus \\
C5 & Nypa \\
C6 & Rhizophora \\
C7 & mucronata \\
C8 & Ceriops \\
C9 & Bruguiera \\
C10 & Sonneratia \\
\hline
\end{tabular}

\subsection{Recognition of Single Mangrove Image}

4.1.1. Recognition of Noisy Images. The samples to be identified are formed by adding noise to the original image, without translation, rotation, or scaling, as shown in Figure 5.

According to the stability analysis method, it can get fast and stable convergent curves when setting network parameters $B=C=1$. The recognition process is shown in Figure 6.

From Figure 7, we can find category $\mathrm{Cl}$ eventually won through the competition. The evolution curve of competitive process could converge quickly and became stable after the 95th iterations.

4.1.2. Recognition of Incomplete Image. The incomplete image to be identified is shown in Figure 8.

The evolution curve is shown in Figure 9.

From Figure 9, we can find the initial order parameter of category $\mathrm{C} 3$ is not the biggest at the beginning (the biggest is C7); however, it eventually won through the competition and desired pattern is recognized. In this process, the category of ambiguous mangrove image will be determined. Meanwhile, the convergence rate of the competitive process could converge quickly and became stable after the 85th iterations.

4.2. Recognition of Large-Scale Images. We take 10 mangrove images as training samples. The size of image is $320 \times 320$. We get 120 test images by various processing methods, such as adding noise, rotation, scaling, cropping, and fuzziness on the training samples. 
$\mathrm{C} 1$

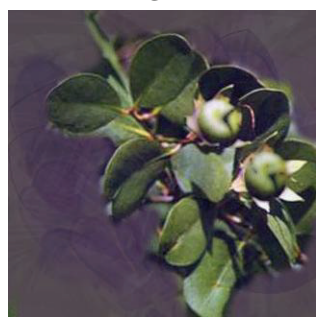

C6

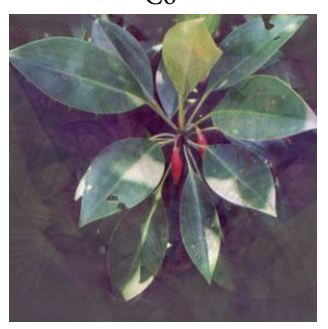

$\mathrm{C} 2$

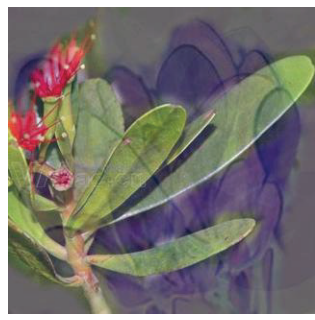

C7

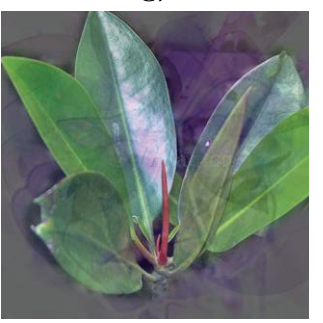

$\mathrm{C} 3$

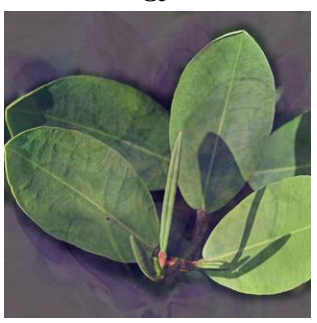

C8

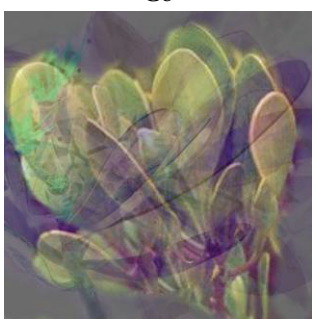

$\mathrm{C} 4$

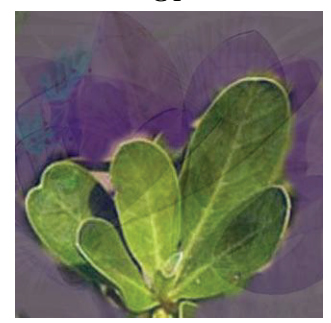

C9

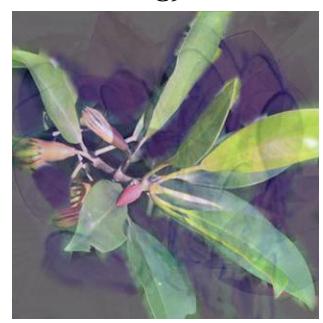

C5

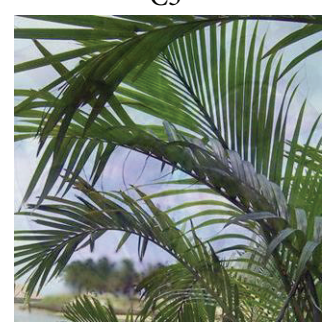

$\mathrm{C} 10$

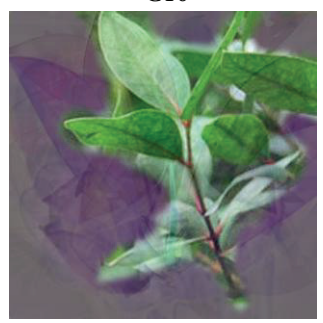

FIGURE 4: The corresponding adjoint pattern.

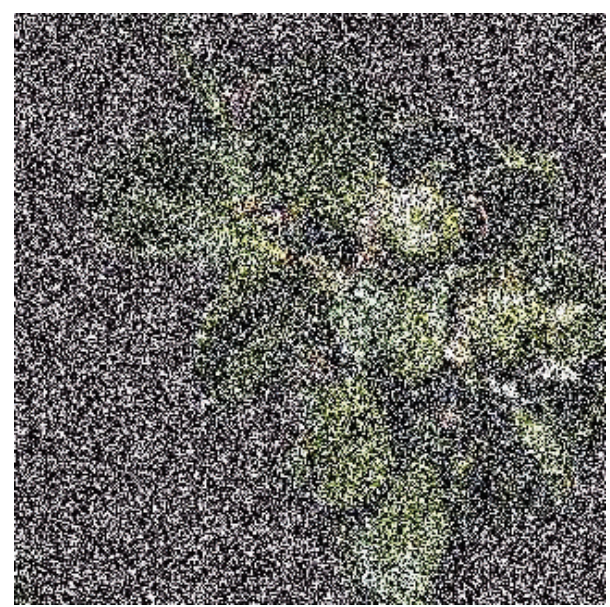

Figure 5: Noisy sample to be identified.

For comparison, we use four strategies:

SNN: mangroves classification based on SNN,

SNN + PSO: mangroves classification based on SNN and PSO,

SNN + QPSO: mangroves classification based on SNN and QPSO,

SNN + AQPSO: mangroves classification based on SNN and AQPSO.

The experiments are implemented in a notebook PC (Lenovo ThinkPad T430) which includes a $2.5 \mathrm{GHz}$ CPU with 4 GB RAM. The operating system is Windows 7.

The parameter setting of PSO is shown as Table 2.

The parameter setting of QPSO and AQPSO is shown as Table 3.

The results are shown in Table 4. Each point is made from average values of over 30 repetitions.
TABLE 2: The parameter setting of PSO.

\begin{tabular}{lcccc}
\hline Algorithm & $\begin{array}{c}\text { Population } \\
\text { size }\end{array}$ & C1 & C2 & $\begin{array}{c}\text { Maximum number } \\
\text { of iterations }\end{array}$ \\
\hline PSO & 30 & 2 & 2 & 20 \\
\hline
\end{tabular}

TABLE 3: The parameter setting of QPSO and AQPSO.

\begin{tabular}{lcccc}
\hline Algorithm & $\begin{array}{c}\text { Population } \\
\text { size }\end{array}$ & $M_{c}$ & $m_{c}$ & $\begin{array}{c}\text { Maximum number } \\
\text { of iterations }\end{array}$ \\
\hline QPSO & 30 & 3 & 0.005 & 20 \\
AQPSO & 30 & 3 & 0.005 & 20 \\
\hline
\end{tabular}

TABLE 4: The testing performance comparison among different optimization methods.

\begin{tabular}{lcccc}
\hline Algorithm & SNN & SNN + PSO & SNN + QPSO & SNN + AQPSO \\
\hline $\begin{array}{l}\text { Accurate } \\
\text { rate (\%) }\end{array}$ & 70.8 & 82.5 & 83.3 & 85.8 \\
Time (s) & 79 & 552 & 528 & 516 \\
\hline
\end{tabular}

TABLE 5: The performances of SNN + QPSO and SNN + AQPSO.

\begin{tabular}{lcccc}
\hline Algorithm & Mean $(\%)$ & SD & Time (s) & Iterations \\
\hline SNN + QPSO & 83.3 & 0.072 & 528 & 12 \\
SNN + AQPSO & 85.8 & 0.052 & 516 & 8 \\
\hline
\end{tabular}

From Tables 4 and 5, we can see no model performs better than others for all evaluation indicators, but the accurate rates of all the three parameter optimization models ( $\mathrm{SNN}+\mathrm{PSO}$, $\mathrm{SNN}+\mathrm{QPSO}$, and SNN + AQPSO) are comparable to SNN model. The reason is that the attention parameters are very important for SNN and optimization algorithm is essential for better performance.

$\mathrm{SNN}+\mathrm{AQPSO}$ can effectively improve accurate rate compared with the standard PSO algorithm and QPSO algorithm. 

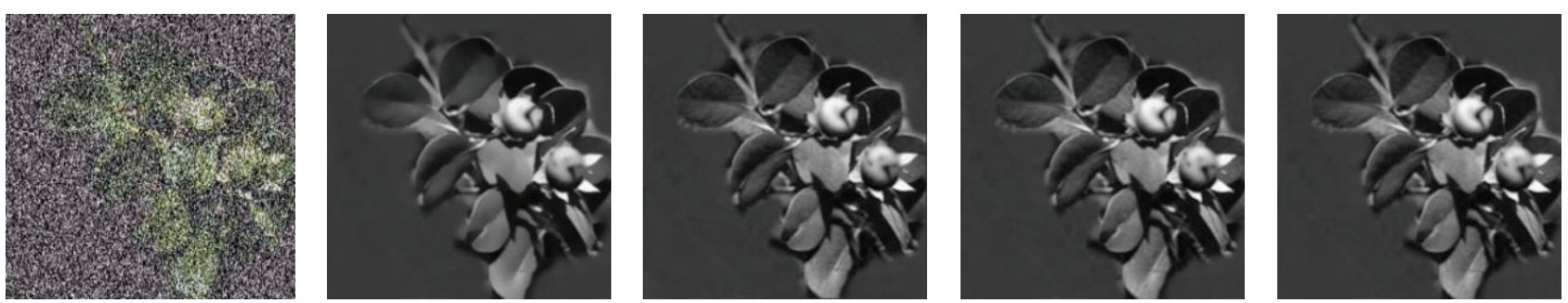

FIgURE 6: The recognition process.

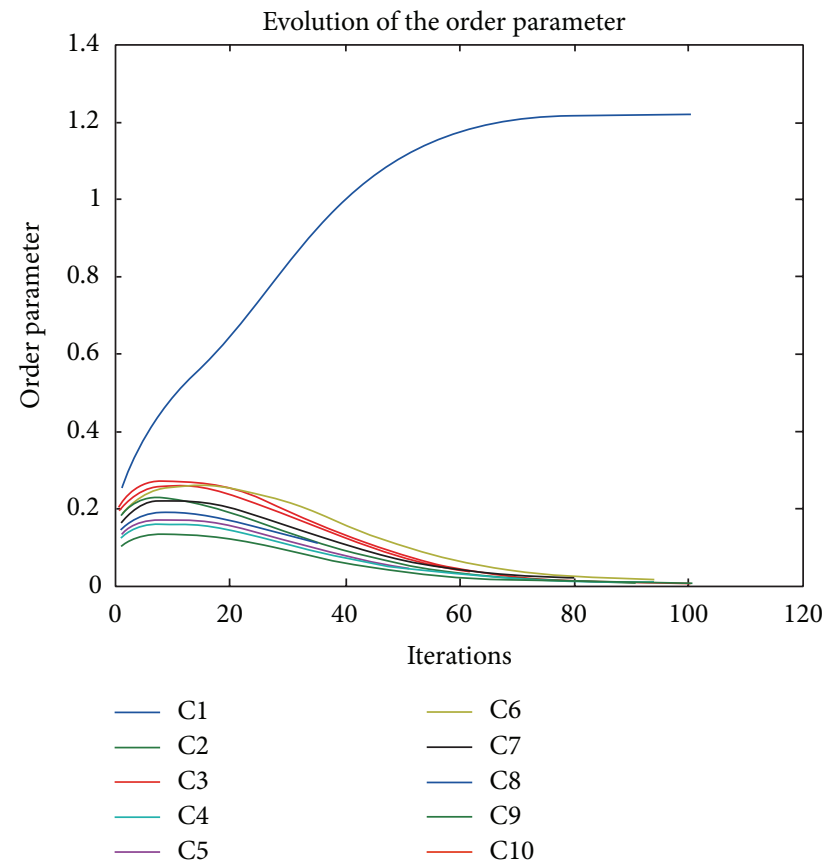

FIGURE 7: The evolution curve of order parameter.

The new behaviors of particle swarm presented in this paper are essential for better performance. At the same time, the behavior selection can enhance decision making ability of behavior selection. On the convergence time, there is a significant improvement as expected. In conclusion, the improved algorithm has better global search ability and fast convergence speed.

The convergence curve is shown in Figure 10. We can see there is a significant improvement as expected on the convergence accuracy. The experiment results show the improved QPSO has better global and local parameter searching abilities.

\section{Conclusions}

In the paper, we construct an improved quantum particle swarm optimization. Experiments on mangroves classification show the improved algorithm has more powerful global exploration ability with faster convergence speed.

We got the following conclusions.

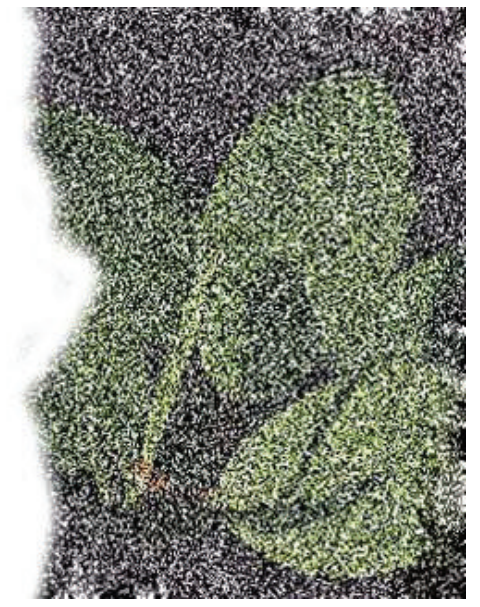

Figure 8: Incomplete sample.

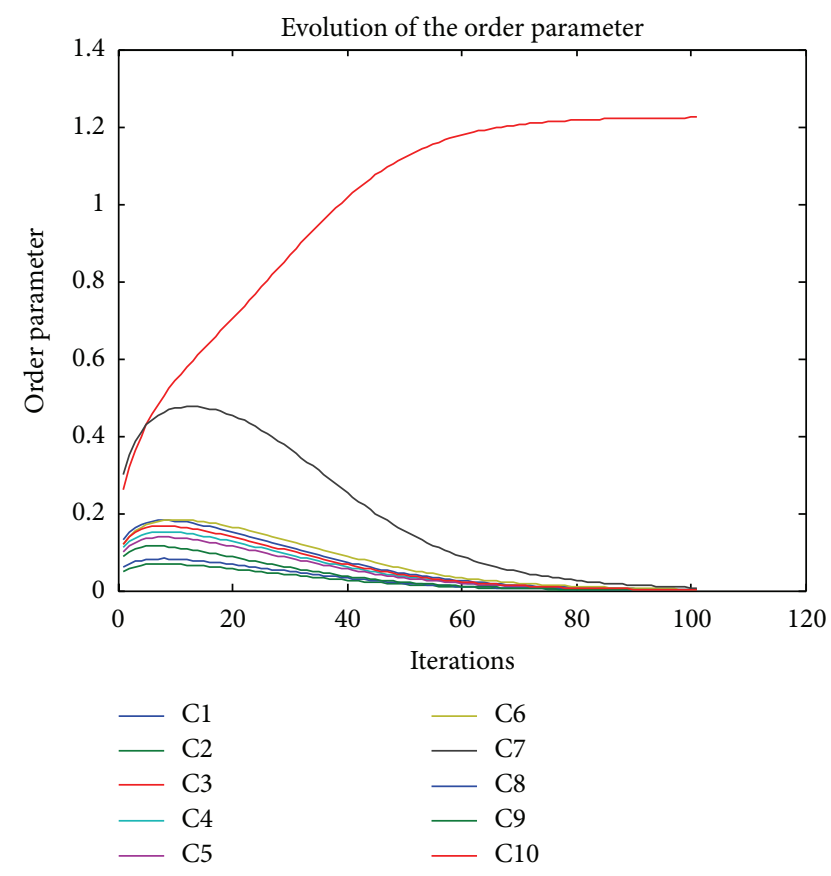

FIGURE 9: The evolution curve of order parameter.

(1) The mangroves classification procedure can be viewed as the competition progress of many order parameters. The order parameter can reflect the similarity between the prototype and test pattern better. 


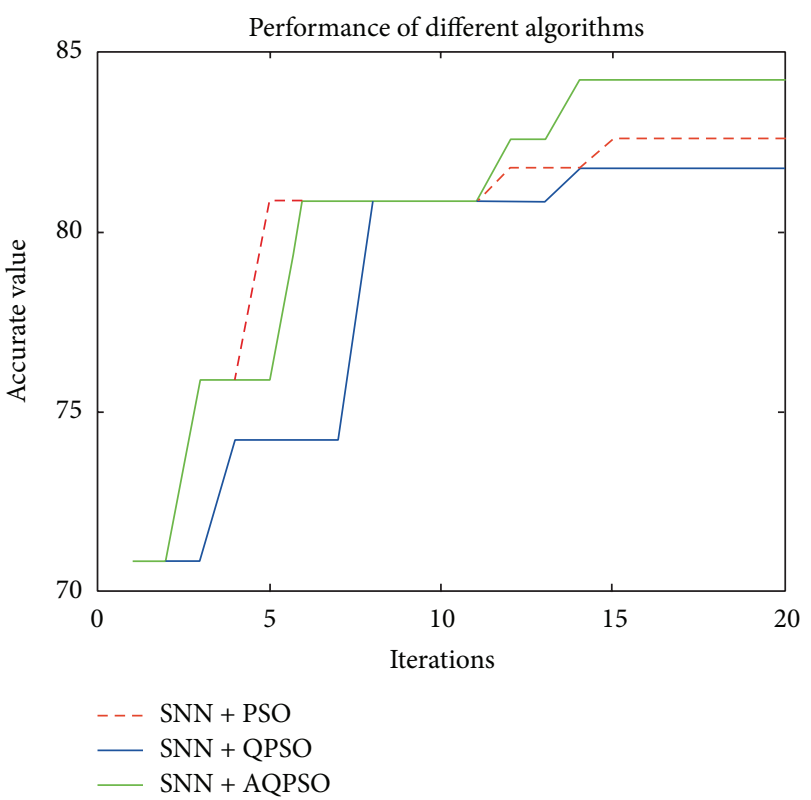

FIGURE 10: Convergence curve.

(2) The AQPSO has both global and local search ability and can effectively choose network parameters of SNN.

In the future, we will focus on the following two aspects.

(1) The network parameters of SNN are very important for better recognition performance. The change of attention parameters will lead to complete different recognition results. We will use other optimization algorithms to search the global optimum parameters of SNN in the corresponding parameter space.

(2) The behavior of particle swarm has a critical influence for better performance. In the future, we will introduce some new behaviors and apply this idea to other optimization tasks.

\section{Conflict of Interests}

The author declares that there is no conflict of interests regarding the publication of this paper.

\section{References}

[1] J. Sun, B. Feng, and W. Xu, "Particle swarm optimization with particles having quantum behavior," in Proceedings of the Congress on Evolutionary Computation (CEC '04), vol. 1, pp. 325-331, June 2004.

[2] P. Moore and G. K. Venayagamoorthy, "Evolving combinational logic circuits using a hybrid quantum evolution and particle swarm inspired algorithm," in Proceedings of the NASA/DoD Conference on Evolvable Hardware (EH '05), pp. 97-102, July 2005.
[3] A. Bagheri, H. Mohammadi Peyhani, and M. Akbari, "Financial forecasting using ANFIS networks with Quantum-behaved Particle Swarm Optimization," Expert Systems with Applications, vol. 41, no. 14, pp. 6235-6250, 2014.

[4] P. Jia, F. Tian, S. Fan, Q. He, J. Feng, and S. X. Yang, "A novel sensor array and classifier optimization method of electronic nose based on enhanced quantum-behaved particle swarm optimization," Sensor Review, vol. 34, no. 3, pp. 304-311, 2014.

[5] H. Ye, W. Luo, and Z. Li, "Convergence analysis of particle swarm optimizer and its improved algorithm based on velocity differential evolution," Computational Intelligence and Neuroscience, vol. 2013, Article ID 384125, 7 pages, 2013.

[6] S. Ch, N. Anand, B. K. Panigrahi, and S. Mathur, "Streamflow forecasting by SVM with quantum behaved particle swarm optimization," Neurocomputing, vol. 101, pp. 18-23, 2013.

[7] M. Jamalipour, R. Sayareh, M. Gharib, F. Khoshahval, and M. R. Karimi, "Quantum behaved Particle Swarm Optimization with Differential Mutation operator applied to WWER-1000 in-core fuel management optimization," Annals of Nuclear Energy, vol. 54, pp. 134-140, 2013.

[8] A. R. Behjat, A. Mustapha, H. Nezamabadi-Pour, M. N. Sulaiman, and N. Mustapha, "Feature subset selection using binary quantum particle swarm optimization for spam detection system," Advanced Science Letters, vol. 20, no. 1, pp. 188-192, 2014.

[9] J. Zhang and L. Shen, "An improved fuzzy c-means clustering algorithm based on shadowed sets and PSO," Computational Intelligence and Neuroscience, vol. 2014, Article ID 368628, 10 pages, 2014.

[10] N. G. Hedeshi and M. S. Abadeh, "Coronary artery disease detection using a fuzzy-boosting PSO approach," Computational Intelligence and Neuroscience, vol. 2014, Article ID 783734, 12 pages, 2014.

[11] J. Sun, W. Xu, and B. Feng, "A global search strategy of quantumbehaved particle swarm optimization," in Proceedings of the IEEE Conference on Cybernetics and Intelligent Systems, pp. 111116, December 2004.

[12] L. D. S. Coelho, "A quantum particle swarm optimizer with chaotic mutation operator," Chaos, Solitons \& Fractals, vol. 37, no. 5, pp. 1409-1418, 2008.

[13] Y. Guofu, "An improved quantum particle swarm optimization algorithm based on real coding method," International Journal of Advancements in Computing Technology, vol. 4, no. 3, pp. 181188, 2012.

[14] D. M. Alongi, "Present state and future of the world's mangrove forests," Environmental Conservation, vol. 29, no. 3, pp. 331-349, 2002.

[15] L. Wei, T. Balz, M. Liao, and L. Zhang, “TerraSAR-X StripMap data interpretation of complex urban scenarios with 3D SAR tomography," Journal of Sensors, vol. 2014, Article ID 386753, 7 pages, 2014.

[16] B. W. Heumann, "Satellite remote sensing of mangrove forests: recent advances and future opportunities," Progress in Physical Geography, vol. 35, no. 1, pp. 87-108, 2011.

[17] C. Giri, E. Ochieng, L. L. Tieszen et al., "Status and distribution of mangrove forests of the world using earth observation satellite data," Global Ecology and Biogeography, vol. 20, no. 1, pp. 154-159, 2011.

[18] R. Jing and L. Kong, "Boundary detection method for largescale coverage holes in wireless sensor network based on minimum critical threshold constraint," Journal of Sensors, vol. 2014, Article ID 985854, 13 pages, 2014. 
[19] Y. Luo, M. Liao, J. Yan, and C. Zhang, "A multi-features fusion support vector machine method (MF-SVM) for classification of mangrove remote sensing image," Journal of Computational Information Systems, vol. 8, no. 1, pp. 323-334, 2012.

[20] V. F. Rodríguez-Galiano, F. Abarca-Hernández, B. Ghimire, M. Chica-Olmo, P. M. Atkinson, and C. Jeganathan, "Incorporating spatial variability measures in land-cover classification using random forest," Procedia Environmental Sciences, vol. 3, pp. 4449, 2011.

[21] H. Haken, Synergetic Cmputers and Cognition: A Top-Down Approach to Neural Nets, Springer, Berlin, Germany, 1991.

[22] J. Shao, J. Gao, and X. Yang, "Synergetic face recognition algorithm based on ICA," in Proceedings of the International Conference on Neural Networks and Brain Proceedings (ICNNB '05), pp. 249-253, Beijing, China, October 2005.

[23] Z. H. Jiang and R. A. Dougal, "Synergetic control of power converters for pulse current charging of advanced batteries from a fuel cell power source," IEEE Transactions on Power Electronics, vol. 19, no. 4, pp. 1140-1150, 2004.

[24] W. Z. Wang, L. Pan, and B. H. Liu, "Synergetic method of traffic state recognition based on manifold learning," in Proceedings of the IEEE International Conference on Automation and Logistics (ICAL '09), pp. 587-591, Shenyang, China, August 2009.

[25] Z. H. Huang and Y. D. Chen, "A two-stage exon recognition model based on synergetic neural network," Computational and Mathematical Methods in Medicine, vol. 2014, Article ID 503132, 7 pages, 2014.

[26] X. L. Ma and L. C. Jiao, "Reconstruction of order parameters based on immunity clonal strategy for image classification," in Image Analysis and Recognition, A. Campilho and M. Kamel, Eds., vol. 3211 of Lecture Notes in Computer Science, pp. 455462, Springer, Berlin, Germany, 2004.

[27] X. L. Ma, S. Wang, and L. C. Jiao, "Robust classification of immunity clonal synergetic network inspired by fuzzy integral," in Advances in Neural Networks-ISNN 2005, J. Wang, X. Liao, and Y. Zhang, Eds., vol. 3497 of Lecture Notes in Computer Science, pp. 26-31, Springer, Berlin, Germany, 2005.

[28] Z. H. Huang and Y. D. Chen, "Log-linear model based behavior selection method for artificial fish swarm algorithm," Computational Intelligence and Neuroscience, vol. 2015, Article ID 685404, 10 pages, 2015.

[29] J. Gao, H. M. Dong, and S. Jing, "Parameters optimization of synergetic recognition approach," Chinese Journal of Electronics, vol. 14, no. 2, pp. 192-197, 2005.

[30] H. L. Wang, The research of application of image recognition using synergetic neural network [Ph.D. thesis], Shanghai Jiao Tong University, Shanghai, China, 2000. 


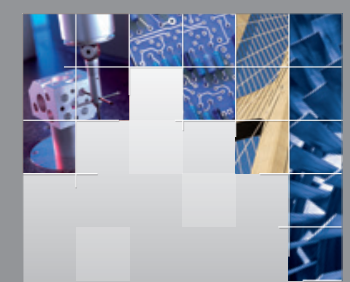

\section{Enfincering}
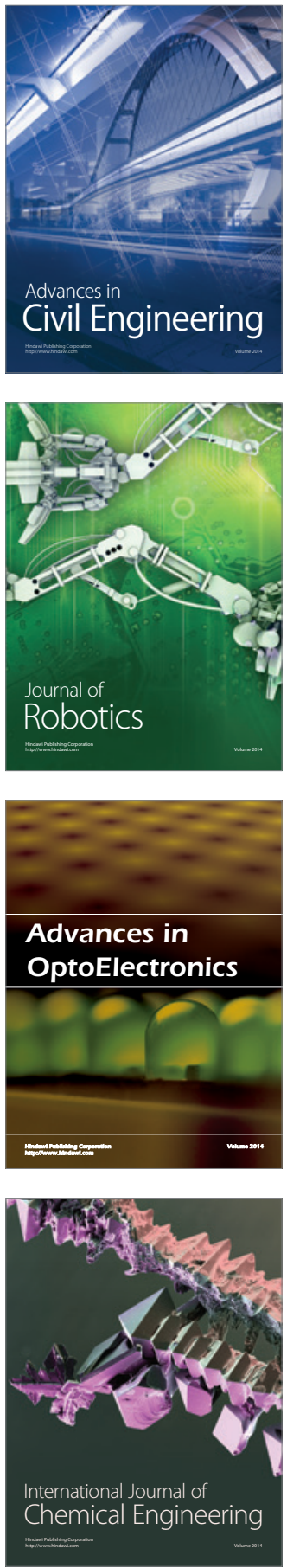

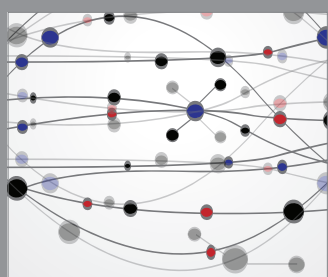

The Scientific World Journal

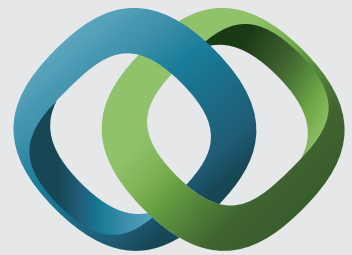

\section{Hindawi}

Submit your manuscripts at

http://www.hindawi.com
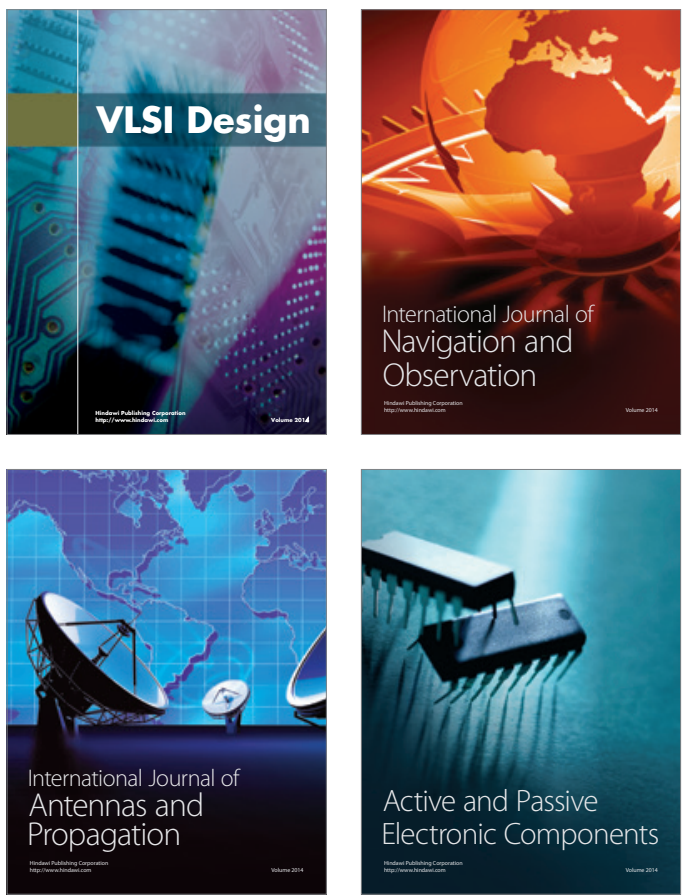
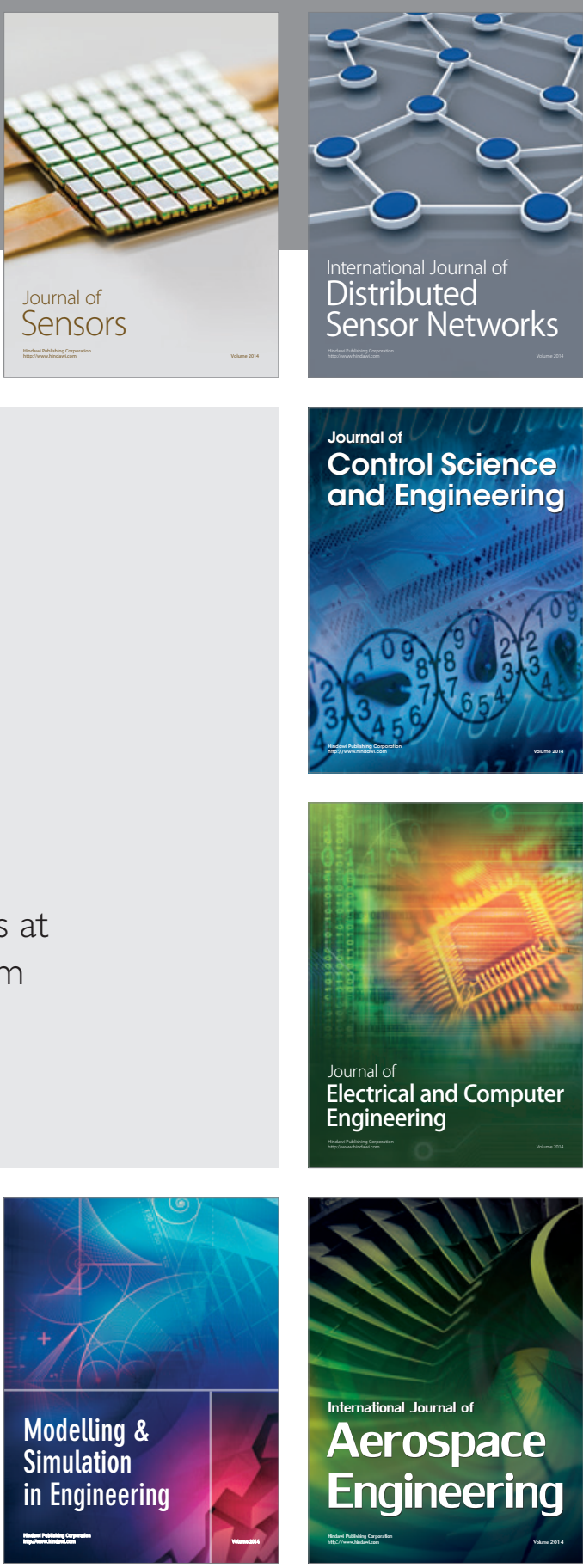

International Journal of

Distributed

Sensor Networks

Journal of

Control Science

and Engineering
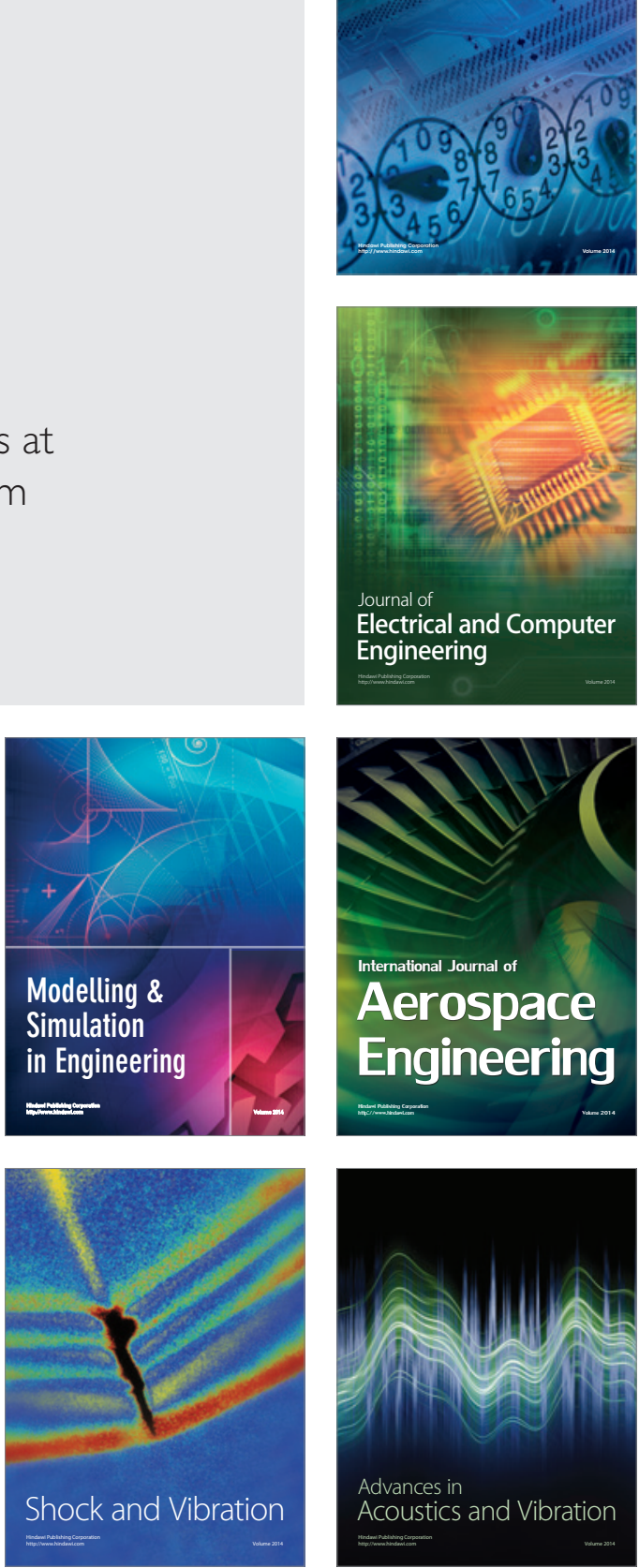